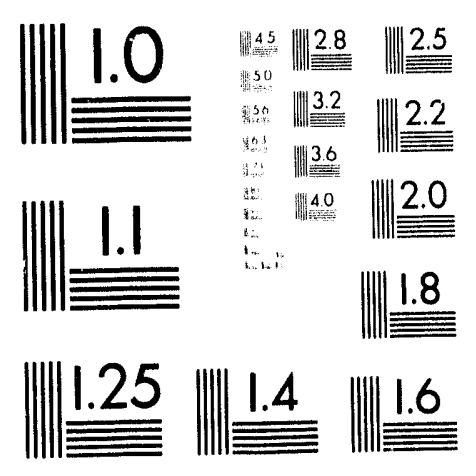



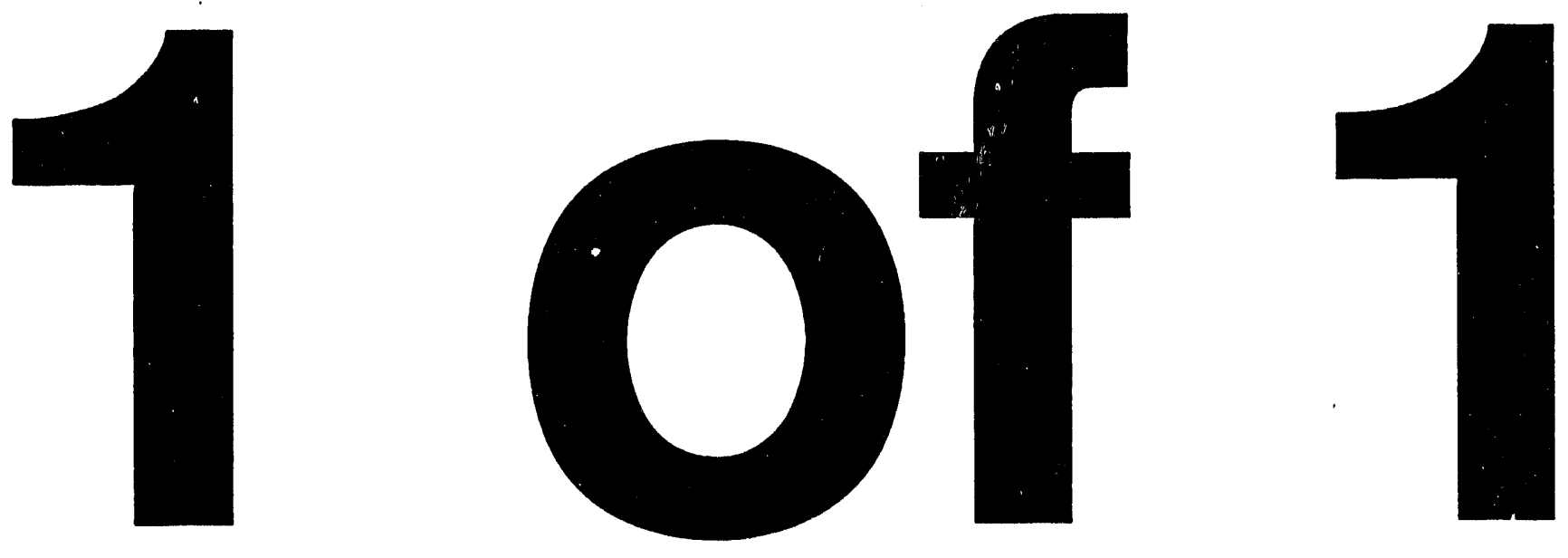


\section{VERIFICATION OF FREQUENCY SCALING LAWS FOR CAPACITIVE RF DISCHARGES USING TWO-DIMENSIONAL SIMULATIONS}

by

V. Vahedi, C. K. Birdsall, M. A. Lieberman, G. DiPeso, and T. D. Rognlien

Memorandum No. UCB/ERL M92/146

15 December 1992

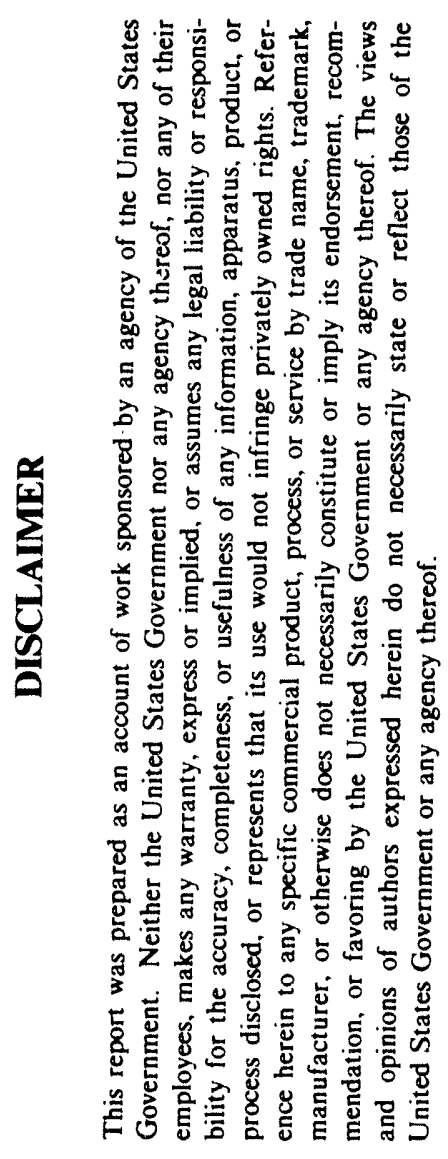

\section{ELECTRONICS RESEARCH LABORATORY}

College of Engineering

University of California, Berkeley

94720 


\title{
Verification of Frequency Scaling Laws for Capacitive RF Discharges Using Two-Dimensional Simulations
}

\author{
V. Vahedi, C. K. Birdsall, and M. A. Lieberman \\ Department of Electrical Engineering and Computer Science \\ and the Electronics Research Laboratory, \\ University of California, Berkeley \\ Berkeley, CA 94720 \\ G. DiPeso and T. D. Rognlien \\ Lawrence Livermore National Laboratory \\ Livermore, CA 94550
}

\begin{abstract}
Weakly ionized processing plasmas are studied in two-dimensions using a bounded particlein-cell (PIC) simulation code with a Monte Carlo Collision (MCC) package. The MCC package models the collisions between charged and neutral particles, which are needed to obtain a self-sustained plasma and the proper electron and ion energy loss mechanisms. A twodimensional capacitive RF discharge is investigated in detail. Simple frequency scaling laws for predicting the behavior of some plasma parameters are derived and then compared with simulation results, finding good agreements. We find that as the drive frequency increases, the sheath width decreases, and the bulk plasma becomes more uniform, leading to a reduction of the ion angular spread at the target and an improvement of ion dose uniformity at the driven electrode.
\end{abstract}




\section{Introduction}

In the last decade, plaunina processing has become an essential step in many manufacturing and engineering areas, ranging from semiconductor processing and very large scale integrated (VLSI) circuit fabrication to hardening of metals and other materials. ${ }^{1}$ Roughly one-third of the steps in state-of-the-art integrated circuit (IC) processing involve plasmas. The role of plasma processing is becoming even more important with decreasing feature sizes on semiconductor wafers. Understanding how and where plasma is created and identifying creation, loss and transport mechanisms for various species in the plasma can assist in the design and production of more efficient plasma sources.

Computer modeling and simulation have proven to be important and effective tools in increasing the general understanding of processing plasmas, giving insights into some discharge parameters which are not easily accessible to laboratory measuring devices. For several years a significant effort has been made to develop self-consistent computer simulation models of self-sustained discharges and plasma. sources. These models typically include interactions between charged particles and electrostatic or electromagnetic fields as well as interactions between charged and neutral particles.

Self-consistent fluid equations have been used by Graves and Jensen, ${ }^{2}$ Boeuf ${ }^{3}$ and Gogolides et al. ${ }^{4}$ to study the structural features of RF and DC glows. These models, however, assume a certain distribution function (typically Maxwellian) for the particles. Since these discharges are inherently complex, and the particle velocity distributions are non-Maxwellian, there has been a considerable effort to develop self-consistent kinetic models without making any assumptions about the distribution functions. Vender, ${ }^{5}$ Surendra et al., ${ }^{6}$ Birdsall, ${ }^{7}$ Vahedi et al. ${ }^{8}$ and Alves et al. ${ }^{9}$ have used Particle-In-Cell (PIC) techniques with the addition of Monte Carlo collisions (MCC) to study RF discharges, and Vahedi et al. ${ }^{10}$ have shown excellent agreement between PIC simulation results and laboratory results by Godyak et al. ${ }^{11} \cdot{ }^{12}$ It should be noted that hybrid (fluid-particle) Monte Carlo modeling of weakly ionized plasmas by Boeuf and Pitchford, ${ }^{13}$ Sato and Tagashira, ${ }^{14}$ and Sommerer and Kushner ${ }^{15}$ has also been successful.

Although in many cases one-dimensional modeling is sufficient to give insights, twodimensional modeling is needed for studying ion flux uniformity across the target and for 
studying density and potential profiles throughout the system. In this work the ion flux $\Gamma_{i}(E, \theta, y)$ at a specific energy $E$ and direction $\theta$ is found as a function of $y$, the dimension along the target electrode. We show by both scaling law arguments and simulations that the ion flux across the target becomes more uniform, directional and mono-energetic as the drive frequency is increased.

\section{PIC-MCC Scheme}

The model we discuss here is a two-dimensional $(x, y)$ bounded electrostatic particle simulation with external circuit elements and an applied uniform or non-uniform magnetic field. A rectangular mesh is placed over the system on which the particle charge densities are accumulated in order to solve for the electrostatic field quantities at the mesh points. The charge-gathering and particle-pushing algorithms used in this model are standard particlein-cell techniques which can be found in Birdsall and Langdon $(1985,1991) .{ }^{16}$ Since the ions are typically much heavier than the electrons and hardly move on the electron time-scale, they are advanced with a much larger time step. This is the essence of electron-field advance and subcycling in particle simulations. ${ }^{16,17}$ Eloctron subcycling improves the efficincy of multiple-species particle simulation by making the cost of advancing the ions neglegible compared with that of the electrons.

In electrostatic codes, Poisson's equation is solved each time-step with the given boundary conditions in order to determine the contribution of the internal charge density and applied voltages to the electric potential inside the system. We use the superposition principle to decompose the field-solve algorithm into Poisson's equation with zero potential boundary conditions and Laplace's equation with the applied boundary conditions where the latter may include external capacitors and multiple RF sources. The separation of the solutions results in a reduced iteration count for the overall field solution. The Laplace equation need onlybe solved initially in order to determine the potential profile due to the external circuit. This approach is very similar to the capacitive matrix method in which the electric potential everywhere in the system is given in terms of the potential at the boundary. ${ }^{18}$

The separation is done by writing

$$
\phi(x, y, t)=\phi_{P}(x, y, t)+\phi_{L}(x, y) V_{L}(y, t)
$$


where $\phi$ is the total electrostatic potential at time $t, \phi_{P}$ is the portion of the total potential due to the charge density inside the device with $\phi_{P}=0$ on the boundaries, and $\phi_{L}$ is the dimensionless potential shape function inside the device due to a specified voltage $V_{L}(y, t)$ on the left boundary, which need not be uniform. Typically, part of the left boundary is grounded and the rest of the left boundary is the target electrode driven by the external circuit. The voltage $V_{L}(y, t)$ is determined by both the external capacitance and the applied RF voltage. The external capacitance stops the flow of the DC current due to the asymmetry of the system (unequal electrode areas. Note that Eq. (1) is only valid when the left boundary contains a driven electrode and the rest of the system is grounded. A more general version of $\mathrm{Eq}$. (1) would include other terms similar to $\phi_{L}(x, y, t) V_{L}(y, t)$ to account for other boundaries containing driven electrodes.

To determine the potential on the driven electrode, we apply Gauss' law as the boundary condition:

$$
\int_{S} \epsilon \mathbf{E} \cdot \mathbf{d} \mathbf{S}=Q
$$

where $Q$, the enclosed charge, is due to the volume charge density $\rho$ inside the enclosed surface $S$ and the wall surface charge density $\sigma$ inside $S$. Drawing a square Gaussian pillbox on a two-dimensional rectangular mesh, as seen in Fig. 1, at the $j^{\text {th }}$ grid on the left boundary (spanning horizontally from $i=-1 / 2$ to $i=1 / 2$ and vertically from $j-1 / 2$ to $j+1 / 2$ ) gives:

$$
\epsilon_{0} E_{x_{1=1 / 2, j}}+\epsilon_{M} E_{y_{1}=0, j+1 / 2} \frac{\Delta x}{\Delta y}-\epsilon_{M} E_{y_{1=0, j-1 / 2}} \frac{\Delta x}{\Delta y}-\epsilon_{M} E_{x_{i=-1 / 2, j}}=\sigma_{j}+\frac{1}{2} \rho_{i=0, j} \Delta x,
$$

where $\sigma_{j}$ is the wall surface charge density, $\rho_{i=0, j}$ is in the volume charge density inside the device due to the plasma at the $j^{\text {th }}$ grid point for $0<j<N_{y}$, and $N_{y}$ is the maximum number of grid points in the $y$ direction. The term $\epsilon_{M}$ in Eq. (3) is the material dielectric constant of the wall. Equation (3) may contain other terms when evaluated at the corner points $\left(j=0\right.$ and $\left.j=N_{y}\right)$. If the left wall is an insulator, the charge density inside the wall is astumed to be lumped in the surface charge density term $\sigma_{j}$.

In this work, we assume that the walls are perfect conductors, in which case the electric field inside the walls $E_{x_{1}=-1 / 2,3}$ and the tangential components $E_{y_{1=0, j+1 / 2}}$ and $E_{y_{t=0, j-1 / 2}}$ are zero every where on the boundaries. We further assume that part of the left boundary (between the grid points $j=N_{1}$ and $j=N_{2}$ ) is driven by the external circuit, and the 
remaining part is grounded along with the other boundaries as seen in Fig. 2. This gives $\phi=0$ as the boundary condition on all the walls except for the driven electrode where

$$
V_{L}(j, t)= \begin{cases}\phi_{0, j} & N_{1} \leq j \leq N_{2} \\ 0 & \text { grounded walls }\end{cases}
$$

Since the driven electrode is assumed to be a perfect conductor, the potential everywhere on the electrode must be the same, i.e. $\phi_{0, j}=\phi_{0}$ for $N_{1} \leq j \leq N_{2}$. The space (which could be several grid points) between the driven electrode and the grounded part of the left boundary on each side (see Fig. 2) is assumed to be filled with vacuum dielectric, and the potential in this gap is linearly interpolated between $\phi_{0}$ and ground.

With these assumptions, Eq. (3) reduces to:

$$
\epsilon_{0} \frac{\phi_{0}-\phi_{1, j}}{\Delta x}=\sigma_{j}+\frac{1}{2} \rho_{i=0, j} \Delta x, \quad N_{1}<j<N_{2},
$$

where Eq. (1) gives $\phi_{1, j}=\phi_{P 1, j}+\phi_{L 1, j} \phi_{0}$. Note that for $j=N_{1}$ and $j=N_{2}$, Eq. (5) contains other terms corresponding to the fields in the vacuum dielectric between the driven electrode and grounded walls. The surface charge density term $\sigma_{j}$ in Eq. (5) is due to the charged particles striking the electrode from the plasma and the charge arriving at the electrode from the external circuit. The total charge density on the driven electrode is then:

$$
\sigma=\sum_{j=N_{1}}^{N_{2}} \sigma_{j} .
$$

The time variation of $\sigma$ is related to the external circuit by:

$$
\frac{d \sigma}{d t}=\frac{I(t)}{A}+J_{p}
$$

where $I(t)$ is the external circuit current, $A$ is the area of the electrode, and $J_{p}$ is the convective current arriving at the electrode due to plasma charged particles. The external circuit seen in Fig. 2 consists of an applied voltage source $V(t)$ and a blocking capacitance $C_{b}$, in which case the external circuit current is given by:

$$
I(t)=C_{b} \frac{d}{d t}\left(V(t)-\phi_{0}\right)
$$

Inserting $\mathrm{Eq}$. (5) into (6) gives an equation for $\sigma$ in terms of the unknown quantity $\phi_{0}$ and sums of known quantities $\phi_{P}, \phi_{L}$, and $\rho$. Combining this new equation for $\sigma$ with Eqs. (7) and $(8)$, we obtain an equation to determine the potential $\phi_{0}$ on the driven electrode. 
To determine the contribution of the internal charge density $\rho$ to the potential, we solve Poisson's equation

$$
\partial_{x}^{2} \phi_{P}+\partial_{y}^{2} \phi_{P}=-\rho / \epsilon_{0}
$$

with the boundary conditions $\phi_{P}=0$ on all boundaries. Methods to solve elliptic equations via finite differences are compared and contrasted in Hockney and Eastwood. ${ }^{18}$ The methods fall into two general classes: iterative techniques and direct matrix inversions. Among the fastest methods is a direct matrix inversion called cyclic reduction. Unlike Fourier transform methods, cyclic reduction can easily be generalized to cylindrical and spherical coordinate systems.

Unfortunately, like Fourier transform methods, cyclic reduction requires the potential equation to be separable. If we want to do implicit particle simulation to gain computational efficiency (longer time steps) while damping plasma oscillations, then we need a method able to solve a modified Poisson's equation given by ${ }^{19}$

$$
\partial_{x}(1+\chi) \partial_{x} \phi_{P}+\partial_{y}(1+\chi) \partial_{y} \phi_{P}=-\tilde{\rho} / \epsilon_{0}
$$

Here the $\chi$ term is the implicit numerical correction to $\phi_{P}$, and $\tilde{\rho}$ is the charge density from particle motion based only on fields known at the present time step. Because $\chi(x, y) \neq$ $f(x) g(y)$ in general, Eq. (10) is not separable and cyclic reduction cannot be used. Instead, we choose a more general, but slower, method to solve Eq. (9) because we want the capability of solving Eq. (10).

The dynamic alternating direction implicit (DADI) method is an iterative technique devised by Doss and Miller ${ }^{20}$ that converges rapidly for problems similar to Eqs. (9) and (10) and is our method of choice. According to Doss and Miller, DADI is only a factor of 4 slower than a fast direct matrix inversion method for solving Eq. (9) and the directional splitting in the scheme makes DADI easily vectorized for large simulations done on vector computers such as the CRAYs. Hewctt, Larson, and Doss ${ }^{21}$ applied a variation of the method to solve a coupled set of elliptic equations arising from a reduced version of Maxwell's equations, and their solutions required much less computer time than the biconjugate gradient method previously used.

DADI works as follows. An artificial time dependence is added to convert Eqs. (9) or (10) into parabolic form, then non-iterative ADI is used to advance the "parabolic" equations in 
"time". A pseudo time step is dynamically adjusted to speed up convergence to the "time" asymptotic state which is the solution of the original elliptic equation. Convergence occurs when the residual of the elliptic equation is less that a chosen tulerance. More detailed explanations can be found in the papers by Doss and Miller ${ }^{20}$ and by Hewett, Larson, and Doss. ${ }^{21}$ Finally, it should be added that for parallel computers, the method of choice may be successive over relaxation (SOR) because although DADI takes fewer iterations to achieve a certain residual, the local nature of SOR requires less communication than an ADI sweep.

We have also added a Monte Carlo collision (MCC) package ${ }^{7},{ }^{22}$ including the null collision method ${ }^{24},{ }^{25}$ to the usual PIC charged particle scheme, as shown in Fig. 3, in order to model collisional plasmas and self-sustained discharges. The full three-dimensional character of a collision is modeled with three velocity components. For our calculations, the neutral particles are assumed to have a Maxwellian velocity distribution and a uniform density between the boundaries. The model remains valid if the neutral density is a function of position and time.

Briefly, the null collision method postulates a fictitious collision such that when its collision frequency is added to the total collision frequency, the "new" total collision frequency becomes constant over the entire energy range. This gives the same collision probability for all the particles independent of the energy. Thus, instead of querying all simulation particles to determine whether a collision has occurred, a certain fraction of all particles is picked at random and tested for collisionality. This method has been shown ${ }^{10}$ to substantially reduce the CPU time for MCC operations.

The MCC package for the argon discharge includes electron-neutral elastic, excitation and ionization collisions as well as ion-neutral scattering and charge-exchange collisions. The electron-neutral cross-sections in the model are the same as those used by Surendra $e t$ $a .^{23}$ as shown in Fig. 4. Figure 5 shows the ion-neutral cross-sections used in the model. ${ }^{26}$

\section{Frequency Scaling Predictions}

We draw upon the work of Misium et al. ${ }^{27}$ to arrive at some simple frequency scaling predictions for capacitive RF discharges. Although the analysis done by Misium et al. is for a one-dimensional system, we assume the same arguments hold in two dimensions as well. 
Throughout the analysis, we assume that the applied voltage is held constant while the RF drive frequency is varied.

Since the applied RF voltage is many times larger than the electron temperature, the sheath width $s$ is much larger than the Debye length $\lambda_{D e}$. Note that because the area ratio of driven to grounded electrodes is typically less than unity, the sheath width and potential drop are larger at the driven (smaller) electrode than at the grounded electrodes. The driven sheath acts as a vacuum capacitor ${ }^{27}$; i.e., there is a displacement current flow $\hat{J}_{D}$ across the sheath which is proportional to

$$
\tilde{J}_{D} \propto \frac{\omega \epsilon_{0}}{s_{D}} \tilde{V}_{D}
$$

where $s_{D}$ is the width of the driven sheath, $\tilde{V}_{D}$ is the RF sheath voltage drop, and $\omega$ is the drive frequency. The subscript $D$ refers to the driven electrode, and the tilded symbols refer to the RF signals.

In capacitive RF discharges, the power gained by the electrons through ohmic and stochastic heatings can be written in the form ${ }^{27}$ :

$$
P_{e l}=K_{o h m}^{\prime} \omega^{2} \tilde{V}_{D}^{1 / 2}+K_{s t o c} \omega^{2} \tilde{V}_{D}
$$

where $K_{o h m}$ and $K_{\text {stoc }}$ are constants which depend only on the electron temperature $T_{e}$ and the gas pressure $p$, independent of the plasma density $n$. The power deposited into the electrons $P_{e l}$ is either lost througiı collisions with the neutrals, $P_{\text {coll }}$, or carried out of the system by the electrons escaping the plasma, $P_{e s c}$ :

$$
P_{e l}=\left(P_{\text {coll }}+P_{e s c}\right) \propto n_{s} u_{B} \mathcal{E}_{L} \propto n,
$$

where $\mathcal{E}_{L}$ is the electron energy loss per ionization event, $u_{B}=\left(k T_{e} / M\right)^{1 / 2}$ is taken to be the ion sound speed at the sheath edge, $n_{s}$ is the ion density at the sheath edge, and $n$ is the average plasma density in the system. At fixed $\dot{V}_{D}$, inserting Eq. (12) into Eq. (13), we see that

$$
P_{e l} \propto n \propto \omega^{2} .
$$

We take the DC ion current $J_{i_{D}}$ through the sheath to have the form of the collisionless Child's law:

$$
J_{i_{D}} \propto \frac{V_{D}^{3 / 2}}{s_{D}^{2}},
$$


where $V_{D}$ is the DC sheath voltage drop at the driven electrode. Assuming the ion current density at the driven sheath edge to be

$$
J_{i_{D}}=n_{s} u_{B} \propto n,
$$

we find from Eq. (15) and Eq. (16) that:

$$
s_{D}^{2} \propto \frac{V_{D}^{3 / 2}}{n}
$$

Assuming $V_{D}$ to be proportional to $\dot{V}_{D}$ for high voltage sheaths, ${ }^{28}$ and since $n \propto \omega^{2}$ from Eq. (14), we see from Eq. (17) that

$$
s_{D} \propto \frac{1}{\omega}
$$

Note that in deriving Eq.(18), if the voltages $\tilde{V}_{D}$ (or $V_{D}$ ) in Eqs. (12) and (17) were retained, the frequency scaling for the sheath width would be

$$
s_{D} \propto \frac{V_{D}^{1 / 2}}{\omega \sqrt{K_{o h m}+K_{s t o c} V_{D}^{1 / 2}}} .
$$

At high pressures, where the ohmic heating is the dominant heating mechanism, this scaling reduces to

$$
s_{D} \propto \frac{V_{D}^{1 / 2}}{\omega} .
$$

However, at low pressures, the stochastic heating becomes the dominant mechanism to accelerate the electrons, and Eq. (19) becomes

$$
s_{D} \propto \frac{V_{D}^{1 / 4}}{\omega} .
$$

Equation (21) suggests that at low pressures, the sheath width is very weakly dependent on the voltage $V_{D}$, which is seen experimentally ${ }^{1229}$ and in computer simulations ${ }^{5}{ }^{6}$

Inserting Eq. (18) into Eq. (11), we arrive at

$$
\tilde{J}_{D} \propto \omega^{2}
$$

If instead of holding $\tilde{V}_{D}$ constant, the power deposited into the system were held constant, then the frequency scaling for the sheath width, the DC sheath voltage drop and the plasma density would be different. Also, if the sheath width is several ion-neutral mean free paths, then the collisional form of Child's law ${ }^{8,30}$ should be used to obtain the frequency scalings, which would give different results. 
The scaling laws predict several advantages of opcrating RF capacitive discharges at higher frequencies including:

- Equation (18) shows that the sheath width decreases as the RF drive frequency increases. This causes the plasma to become more uniform along the target.

- As the sheath width decreases and becomes comparable to the ion-neutral mean free path $\lambda_{\text {in }}$, the ion motion in the sheath becomes less collisional, causing the ions to arrive more nearly normal to the target. The decrease in the ion angular spread is desirable to avoid undercut in etching. ${ }^{1}$

- Equation (12) suggests that at a fixed input power, the DC sheath voltage drop, $V_{D} \approx \tilde{V}_{D}$, decreases as the drive frequency increases. Since the ions only respond to the time averaged potential in the system, as the sheath voltage drops, the ion impacting energy at the target decreases, causing less damage to the target.

Surendra et al..$^{31}$ used a particle-in-cell simulation code to investigate the frequency dependence of various plasma parameters in a one-dimensional system and obtained favorable results similar to these scaling laws. Also, the ion flux uniformity improvement at higher frequencies was observed experimentally by Howling $e t a l^{32}$ in a parallel plate capacitive RF discharge.

\section{Capacitive RF Discharge Simulations}

The simulated system is shown in Fig. 2. As discussed before, the space between the grounded and the diven electrode is assumed to be a vacuum dielectric of a few simulation grid cells. This gap induces large fringing electric fields which can accelerate extracted ions to large bombarding energies. The dimensions of the system are set to be $L_{x}=4 \mathrm{~cm}$,

$L_{y}=5 \mathrm{~cm}, L_{\text {electrode }}=3 \mathrm{~cm}$. Although the simulation is uniform and infinite in the $z$ dimension, the height of the system is arbitrarily chosen to be $100 \mathrm{~cm}$ to give the system a specific volume needed for such quantities as the charge density. With these dimensions, the area of the driven electrode is $300 \mathrm{~cm}^{2}$, and the system capacitance withrut the plasma is $C_{s_{s} s_{0}} \sim 20 \mathrm{pF}$. The blocking capacitance in the external circuit was chosen to be $C_{b}=500$ 
$\mathrm{pF}$, an order of magnitude larger than $C_{s y s_{0}}$ so that it would have a negligible impedance at the RF driving frequency. This blocking capacitance, as discussed before, stops the flow of DC current in the system. As the blocking capacitor charges up, a negative DC self-bias $V_{\text {bias }}$ appears at the driving electrode which is characteristic of asymmetric RF capacitive discharges. Because the other boundaries are grounded, and the average plasma potential $V_{p}$ stays positive with respect to the ground, the plasma-to-driven-electrode voltage $V_{D}$ (the ion bombarding energy) is given by:

$$
V_{D}=-V_{b i a s}+V_{p}
$$

The RF voltage $\tilde{V}_{D}$ that appears at the driving electrode is found from:

$$
\tilde{V}_{D}=\tilde{V}_{0} \frac{C_{b}}{C_{b}+C_{\text {sys }}}
$$

where $\tilde{V}_{0}$ is the magnitude of the voltage source, and $C_{s y s}$ is the capacitance of the system with the plasma.

Three cases were studied for the drive frequencies of 11,30 , and $50 \mathrm{MHz}$ to check the frequency scalings of Eqs. (14), (18) and (22). The applied voltage $\dot{V}_{0}$ was held at $200 \mathrm{~V}$ and the neutral gas pressure was fixed at $10 \mathrm{mTorr}$. As we can see in Fig. 6, the sheath width measured at the driven electrode, $s_{D}$, is indeed inversely proportional to the drive frequency. As the sheath width decreases, the capacitance of the system $C_{\text {sys }}$ increases, which in turn causes the RF voltage at the driven electrode to decrease as predicted by Eq. (24). This was also seen in the simulations as shown in Fig. 7. However, if the blocking capacitance is large enough, this effect can be ignored and the applied voltage assumed to be roughly constant as was done in deriving the scaling predictions. Figure 7 also shows that as the RF drive frequency increases, the plasma potential $V_{p}$ decreases, while the magnitude of the negative DC bias $V_{b i a s}$ at the driven electrode increases. The DC plasma-to-driven-electrode voltage $V_{D}$ obtained from Eq. (23), however, appears to remain roughly constant over the three RF driv frequencies in Fig. 7 , consistent with the high voltage sheath law that $V_{D} \approx \tilde{V}_{D}$, with $\tilde{V}_{D}$ held approximately fixed in the simulations.

The power deposited into the system, the plasma density, and the AC current density are plotted logarithmically in Figs. 8, 9, and 10 respectively. They roughly show quadratic dependence on the drive frequency as predicted by Eqs. (14) and (22). The deviation 
from the quadratic scaling can perhaps be attributed to the two-dimensional nature of the discharge. Recall that the Misium's model ${ }^{27}$ is derived for a one-dimensional system with a fixed volume. In our model, as the drive frequency increases the sheaths become thinner, and the plasma volume changes drastically, which can certainly affect the scaling laws. Figures 11-13 show potential profiles in the system at drive frequencies of 11,30 , and $50 \mathrm{MHz}$, respectively. As the drive frequency increases, the sheath width drops, as predicted by Eq. (18), and the bulk plasma extends through the system, causing the plasma to become more uniform over the driven electrode.

The ion dose at the target can be obtained numerically by integrating the ion flux over a time interval. Figure 14 shows the normalized ion dose versus $y$ at the target at 11, 30, and $50 \mathrm{MHz}$ frequencies. The peaks at the corners of the profile correspond to the large number of ions accelerated by the fringing fields generated around the gap. These peaks are seen at the edge of the driven electrode in all three cases. At the $11 \mathrm{MHz}$ drive frequency, the ion dose in the middle of the driven electrode is greater than the dose close to the edge of the electrode. This non-uniformity in the ion dose can lead to non-uniform etching which is undesirable. There is also a more rapid variation on the scale of a few millimeters which is due to the statistical variation from the finite number of simulation particles. Figure 14 shows that at higher frequencies, the overall profile flattens. Thus, the ion dose uniformity improves at higher frequencies.

As seen in Fig. 6, the sheath width decreases as the drive frequency increases, which causes the ion motion in the sheath to be less collisional. As the ion-neutral collisionality in the sheath drops, more ions arrive at the target with the energy corresponding to the DC sheath voltage drop. This causes the ion energy distribution at the target to become more mono-energetic as seen in Figs. 15-17. Because the ions make fewer collisions in the sheath as the sheath width becomes comparable to the ion-neutral mean free path, the ion motion becomes more directed toward the target, which decreases the ion angular spread at the arget. This is seen in Fig. 18 which depicts the average ion angle at the target as a function of $y$. The peaks seen in Fig. 18 are generated by the ions accelerated by the fringing fields around the gap. The average ion angle at the edges of the driven electrode is roughly 20 degrees in the three cases, but the angular spread in the middle of the target decreases from 5.1 degrees at $11 \mathrm{MHz}$ to 2.3 degrees at $50 \mathrm{MHz}$. It can also be seen from Fig. 18 that 
as the drive frequency increases, the fraction of the driven electrode over which the angular spread remains constant increases. This increase improves the directionality and uniformity, both of which are desirable in reactive ion etching (RIE) processes where a vertical etch is needed to avoid undercut. ${ }^{1}$

\section{Conclusions}

We have developed a bounded two dimensional particle-in-cell simulation code with a Monte Carlo Collision (MCC) package to study processing discharges. The pressure and the applied voltage were held constant while the frequency was varied from 11 to $50 \mathrm{MHz}$ to check the predicted frequency scalings of the sheath width, the plasma density, and power deposited into the electrons. The frequency scaling of the various parameters was verified.

Simulation of two-dimensional capacitive discharges suggests that operating RF disrnarges at higher frequencies has several advantages, including the improvement of the ion dose uniformity at the driven electrode. At higher frequencies the sheath width decreases and the ion motion in the sheath becomes less collisional, causing the ions to arrive normal to the target with a mono-energetic distribution and less angular spread.

\section{Acknowledgment}

This work was performed with support in part from the Lawrence Livermore National Laboratory under U.S. Department of Energy Contract W-7405-ENG-48. The portion of the work was performed in Berkeley was supported by the National Science Foundation under Grant ECS-8910827, U.S. Department of Energy Contract DE-FG03-90ER54079, and Office of Naval Research Contract FD-N00014-90-J-1198. The authors greatly appreciate the help, support, and input of D. W. Hewett and R. H. Cohen. 


\section{References}

${ }^{1}$ D. M. Manos and D. L. Flamm, Plasma Etching, Academic Press (1989).

${ }^{2}$ D. B. Graves and K. F. Jensen, IEEE Trans. Plasma Sci. PS-14, 78 (1986).

${ }^{3}$ J. P. Boeuf, Phys. Rev. A 36, 2782 (1987).

${ }^{4}$ E. Gogolides, J. P. Nicolai, and H. H. Sawin, J. Vac. Sci. Technol. A 7, 1001 (1989).

${ }^{5}$ Vender D., and R. W. Boswell, IEEE Trans. Plasma Sci. 18, 725 (1990).

${ }^{6}$ Surendra M., and D. B. Graves, IEEE Trans. Plasma Sci. 19, 144 (1991).

${ }^{7}$ Birdsall C. K., IEEE Trans. Plasma Sci. 19, 65 (1991).

${ }^{8}$ V. Vahedi, M. A. Lieberman, M. V. Alves, J. P. Verboncoeur, and C. K. Birdsall, J. Appl. Phys. 69, 2008 (1991).

${ }^{9}$ M. A. Alves, M. A. Lieberman, V. Vahedi, and C. K. Birdsall, J. Appl. Phys., 69, 3823 (1991).

${ }^{10}$ V. Vahedi, M. Surendra, G. DiPeso, P. Mirrashidi, and C. K. Birdsall, 1992 Int'l Conf. on Plasma Physics, III-1993, Innsbruck, Austria (1992).

${ }^{11}$ V. A. Godyak and R. B. Piejak, Phys. Rev. Lett. 65, 996 (1990).

${ }^{12}$ V. A. Godyak, R. B. Piejak and B. M. Alexandrovich, IEEE Trans. Plasma Sci., 19, 660 (1991).

${ }^{13}$ J. P. Boeuf and L. C. Pitchford, IEEE Trans. Plasma Sci 19, 286 (1991).

${ }^{14}$ N. Sato and H. Tagashira, IEEE Trans. Plasma Sci 19, 102 (1991).

${ }^{15}$ T J. Sommerer and M. J. Kushner, J. Appl. Phys. 711654 (1992).

${ }^{16}$ Birdsall C. K. and A. B. Langdon, Plasma Physics Via Computer Simulation, Adam Hilger, Bristol, Philadelphia and New York (1991).

${ }^{17}$ J. C. Adam, A. Gourdin-Serveniere, and A. B. Langdon, J. Comp. Phys. 47, 229 (1982). 
${ }^{18}$ R. W. Hockney and J. W. Eastwood, Computer Simulation Using Particles, Adam Hilger, Bristol and Philadelphia (1988).

${ }^{19} \mathrm{~A}$. B. Langdon and D. C. Barnes, in Multiple Time Scales, editted by J. Brackbill and B. 1. Cohen, Academic Press Inc., Orlando (1985).

${ }^{20}$ S. K. Doss and K. A. Miller, Siam J. Numer. Anal. 16, 837 (1979).

${ }^{21}$ D. W. Hewett, D. J. Larson, and S. K. Doss, J. Comp. Phys. 101, 11 (1992).

${ }^{22}$ M. Surendra, D. B. Graves, and I. J. Morey, Appl. Phys. Lett. 56, 1022 (1990).

${ }^{23}$ M. Surendra, D. B. Graves, and G. M. Jellum, Phys. Rev. A 41, 1012 (1990).

${ }^{24}$ Y. Kaufman, J. Phys. D 21, 442 (1988).

${ }^{25}$ S. L. Lin and J. N. Bardsley, J. Chem. Phys. 66, 435 (1977).

${ }^{26}$ W. H. Cramer, J. Chem. Phys. 30, 641 (1959).

${ }^{27}$ G. R. Misium, A. J. Lichtenberg, and M. A. Lieberman, J. Vac. Sci. Technol. A 7, 3 (1989).

${ }^{28}$ V. A. Godyak and N. Sternberg, Phys. Rev. A 422299 (1990).

${ }^{29}$ N. Mutsukura, K. Kobayashi, and Y. Machi, J. Appl. Phys. 68, 2657 (1990).

${ }^{30}$ M. A. Lieberman, J. Appl. Phys. 65, 4186 (1989).

${ }^{31}$ M. Surendra, D. B. Graves, Appl. Phys. Lett. 59, 2091 (1991).

${ }^{32}$ A. A. Howling. J. L Dorier, C. Hollenstein, U. Kroll, J. Vac. Sci. Technol. A, 1080 (1992). 


\section{List of Figures}

1 The Gaussian pillbox, centered at the $j^{\text {th }}$ grid, used to determine the boundary condition on the left boundary. Note that if the wall is assumed to be a perfect conductor, the $\mathrm{e}^{\mathrm{s}}$ sctric field inside and the tangential components of the electric field on the wall must be zero. In case of an insulator, the tangential fields and the electric field inside the wall can be non-zero. . . . .

2 The two-dimensional simulated system. All the boundaries are grounded except for part of the left boundary, which is the driven electrode. The external circuit consists of a voltage source and a blocking capacitor which stops the flow of the DC current. . . . . . . . . . . . . . . . . . . . . . 19

3 The flow chart for an explicit PIC scheme with the addition of the Monte Carlo collision package, called PIC-MCC. . . . . . . . .

4 The argon electron-neutral cross-sections used in this model. . . . . . . . . . 21

5 The argon ion-neutral cross-sections used in this model. . . . . . . . . . . 22

6 The logarithmic plot of sheath width $s_{D}$ at the driven electrode versus the $\mathrm{RF}$ drive frequency. The sheath width is inversely proportional to the drive frequency as seen in this plot. .................

7 Frequency scalings for the RF voltage that appears at the driven electrode, $V_{a c}\left(\right.$ or $\tilde{V}_{D}$ ), the plasma potential $V_{p}$, and the magnitude of the negative DC bias at the driven electrode $V_{b i a s}$. As the RF drive frequency increases, $V_{a c}$, and $V_{p}$ both decrease, while the magnitude of $V_{\text {bias }}$ increases. However, the DC plasma-to-driven-electrode voltage $V_{D}$ obtained from Eq. (23) stays the same for the three drive frequencies. . . . . . . . . . . . . . . .

8. The logarithmic plot of the power $P_{\text {in }}$ deposited into the system. versus the RF drive frequency. The power scales as nearly frequency squared. . . . . . 25

9 The logarithmic plot of the plasma density $n$ versus the RF drive frequency. The plasma density, is proportional to the power, which also scales nearly as frequency squared. 
10 The logarithmic plot of the RF current $\tilde{J}_{D}$ flowing in the external circuit versus the RF drive frequency. The RF current is nearly proportional to the

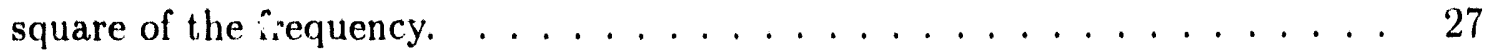

11 The time-averaged potential profile $\phi(x, y)$ in the system at the drive frequency of $11 \mathrm{MHz} \ldots \ldots \ldots \ldots \ldots$

12 The time-averaged potential profile $\phi(x, y)$ in the system at the drive frequency of $30 \mathrm{MHz} \ldots \ldots \ldots \ldots \ldots \ldots \ldots$

13 The time-averaged potential profile $\phi(x, y)$ in the system at the drive frequency of $50 \mathrm{MHz} \ldots \ldots \ldots \ldots$. . . . . . . . . . . . . . . . . . . . . . . . . .

14 The normalized ion dose at the target versus $y$ at frequencies of 11,30 , and $50 \mathrm{MHz}$. The dark rectangle at the bottom of each plot corresponds to the driven electrode and the light rectangles correspond to the grounded electrodes. 31

15 The normalized ion flux energy distribution at the driven electrode at the drive frequency of $11 \mathrm{MHz} \ldots \ldots \ldots$. . . . . . . . . . . . . . 32

16 The normalized ion flux energy distribution at the driven electrode at the drive frequency of $30 \mathrm{MHz} \ldots \ldots \ldots 33$

17 The normalized ion flux energy distribution at the driven electrode at the drive frequency of $50 \mathrm{MHz} \ldots \ldots \ldots \ldots$. . . . . . . . . . . . 34

18 The average ion angle at the target versus $y$ at frequencies of 11,30 , and 50 $\mathrm{MHz}$. The dark rectangle at the bottom of each plot corresponds to the driven electrode and the light rectangles correspond to the grounded electrodes. . 


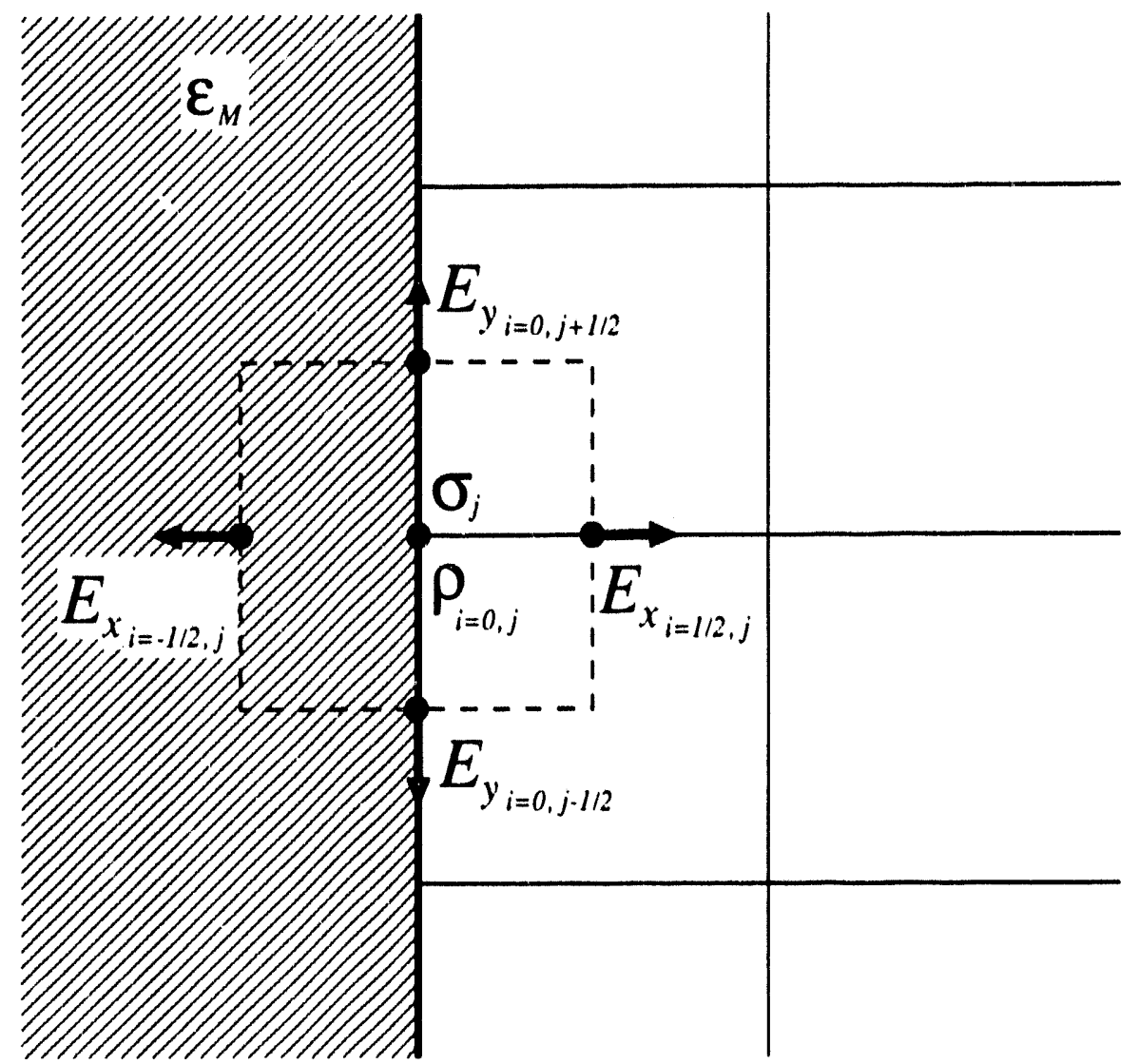

Figure 1: 


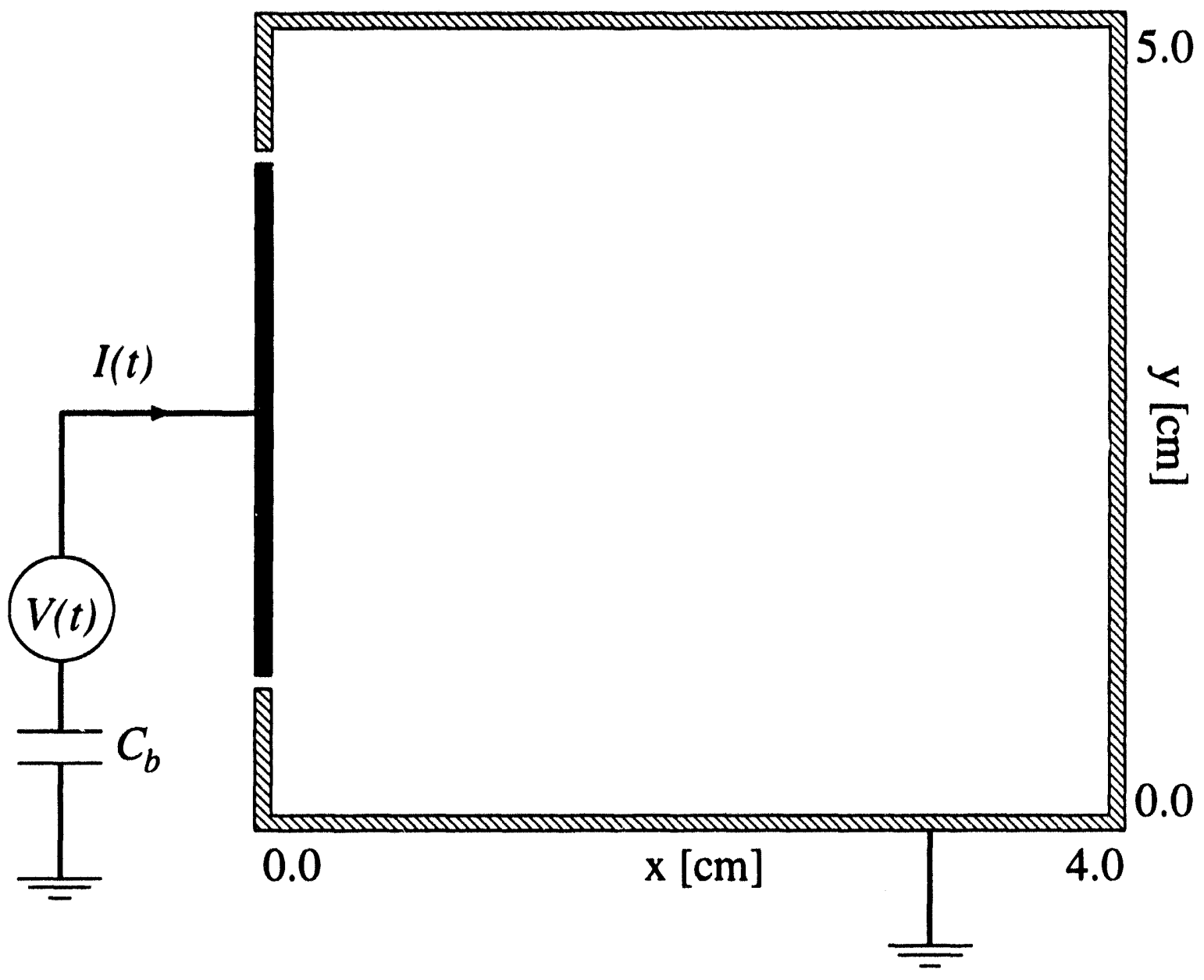

Figure 2: 


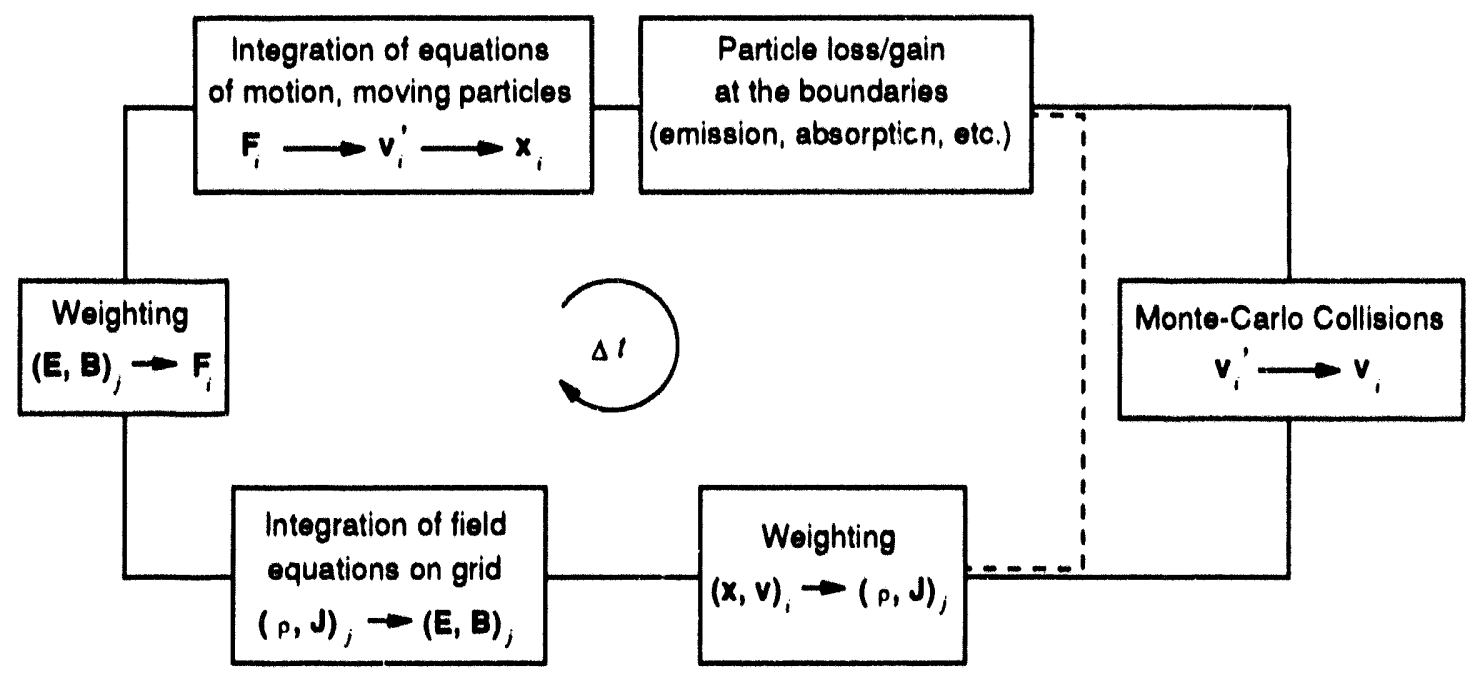

Figure 3: 


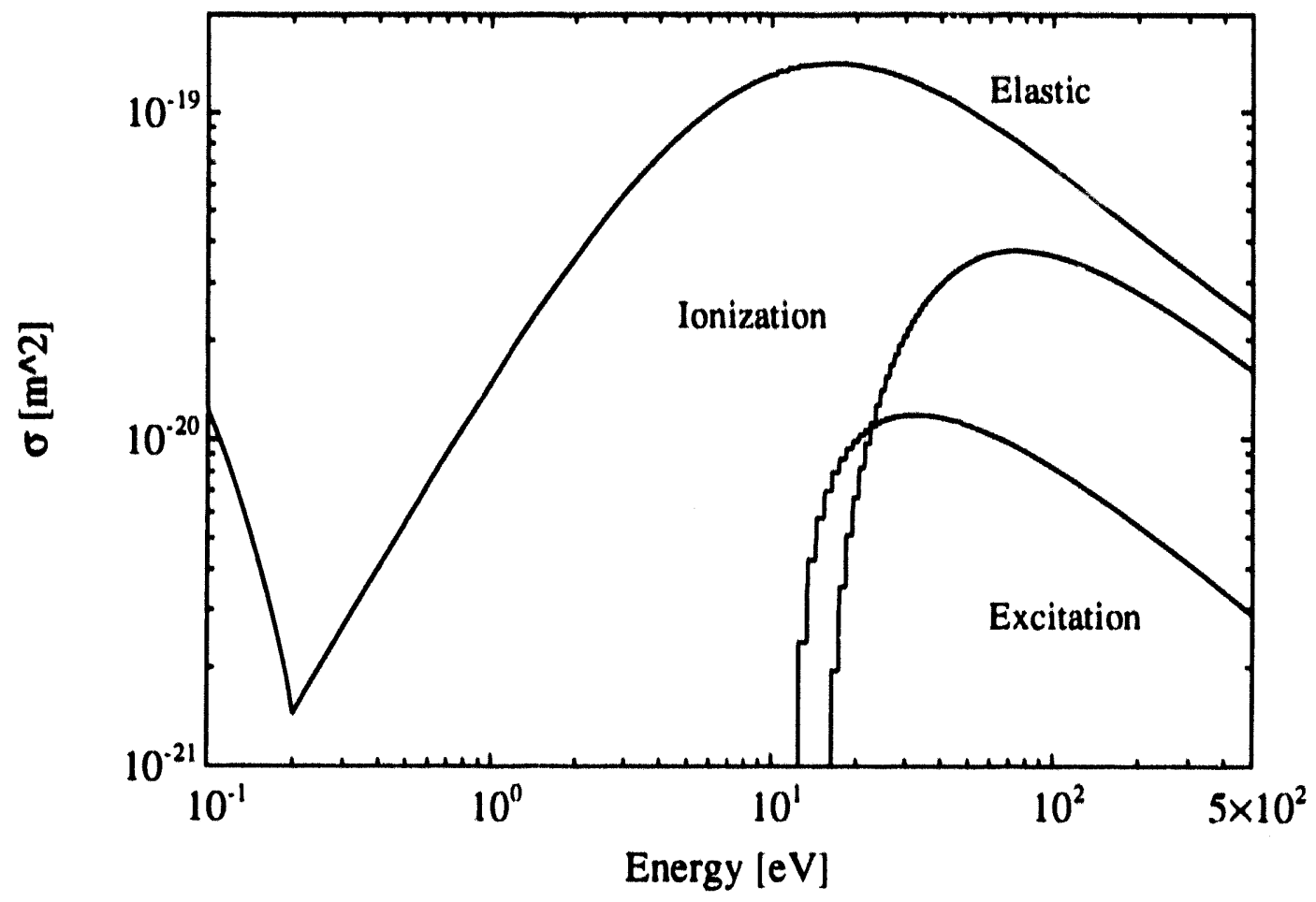

Figure 4: 


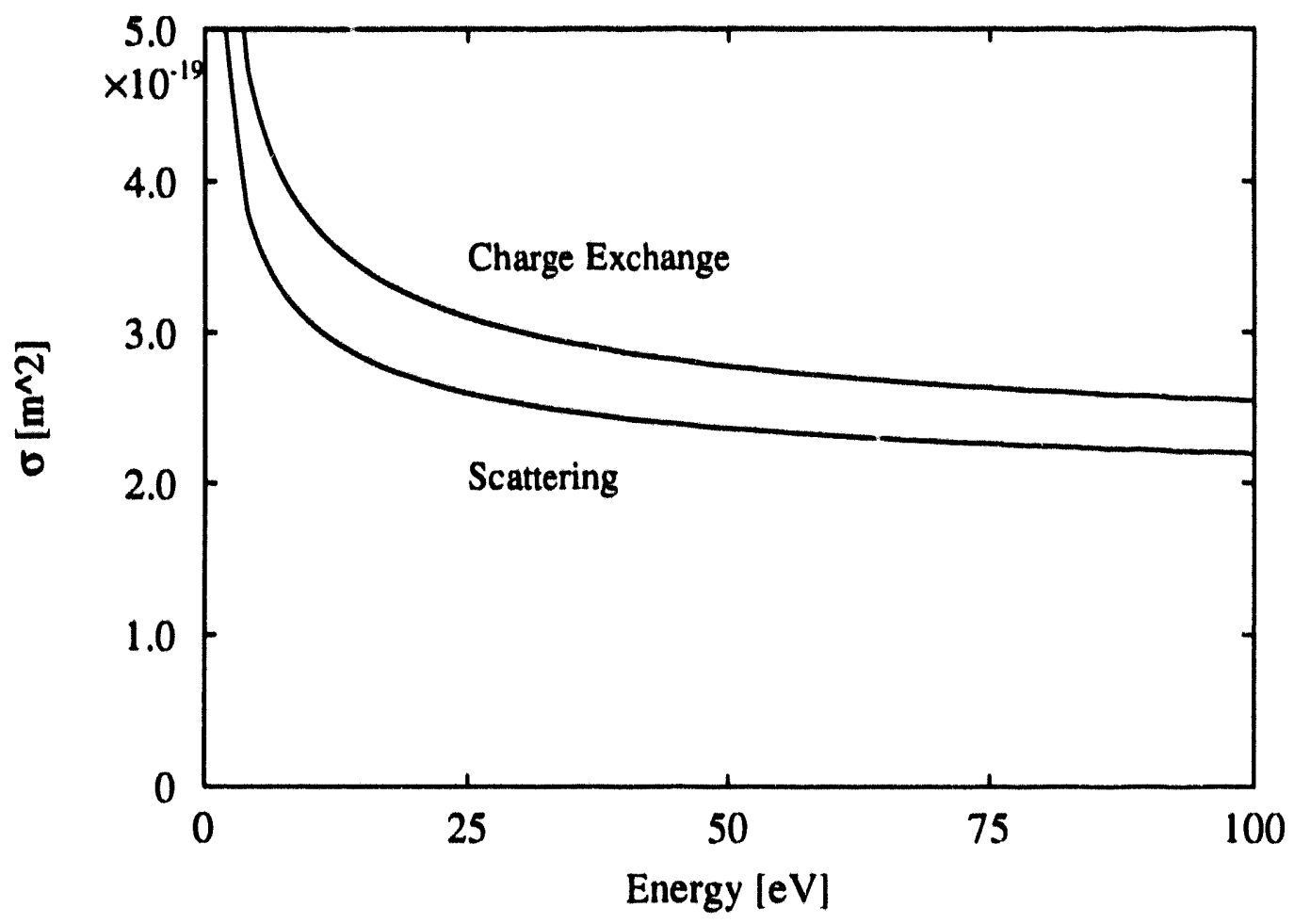

Figure 5: 


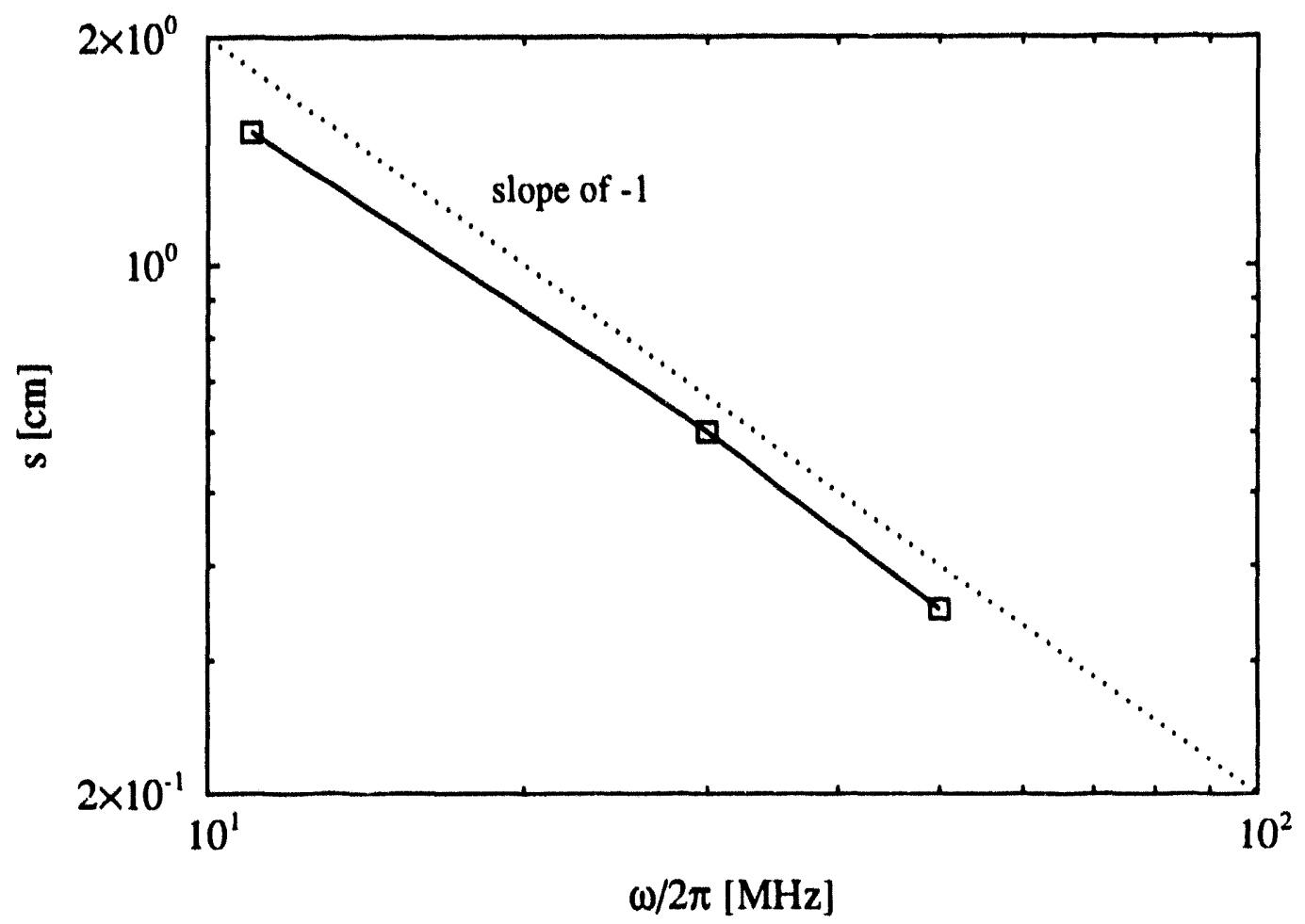

Figure 6: 


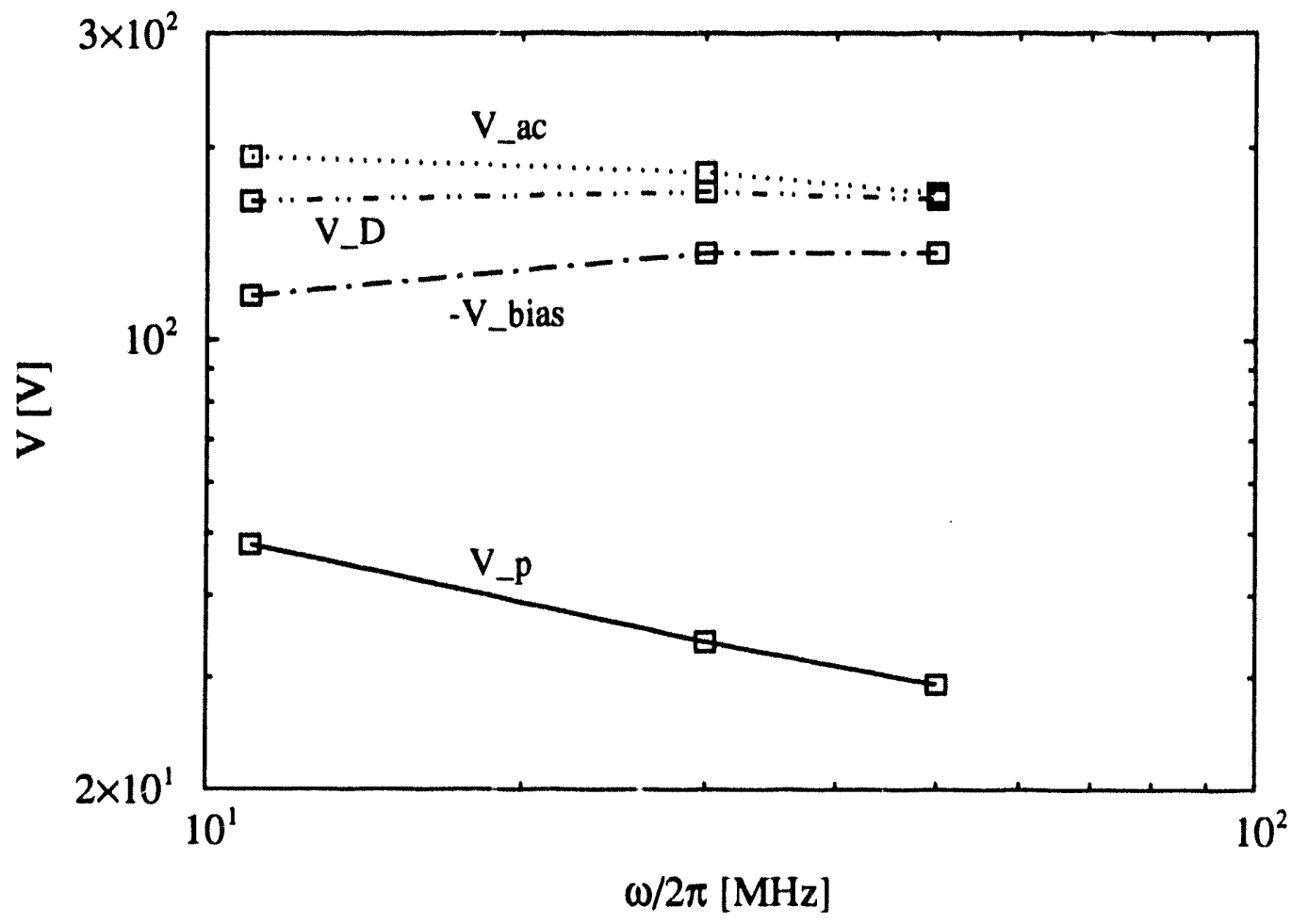

Figure 7: 


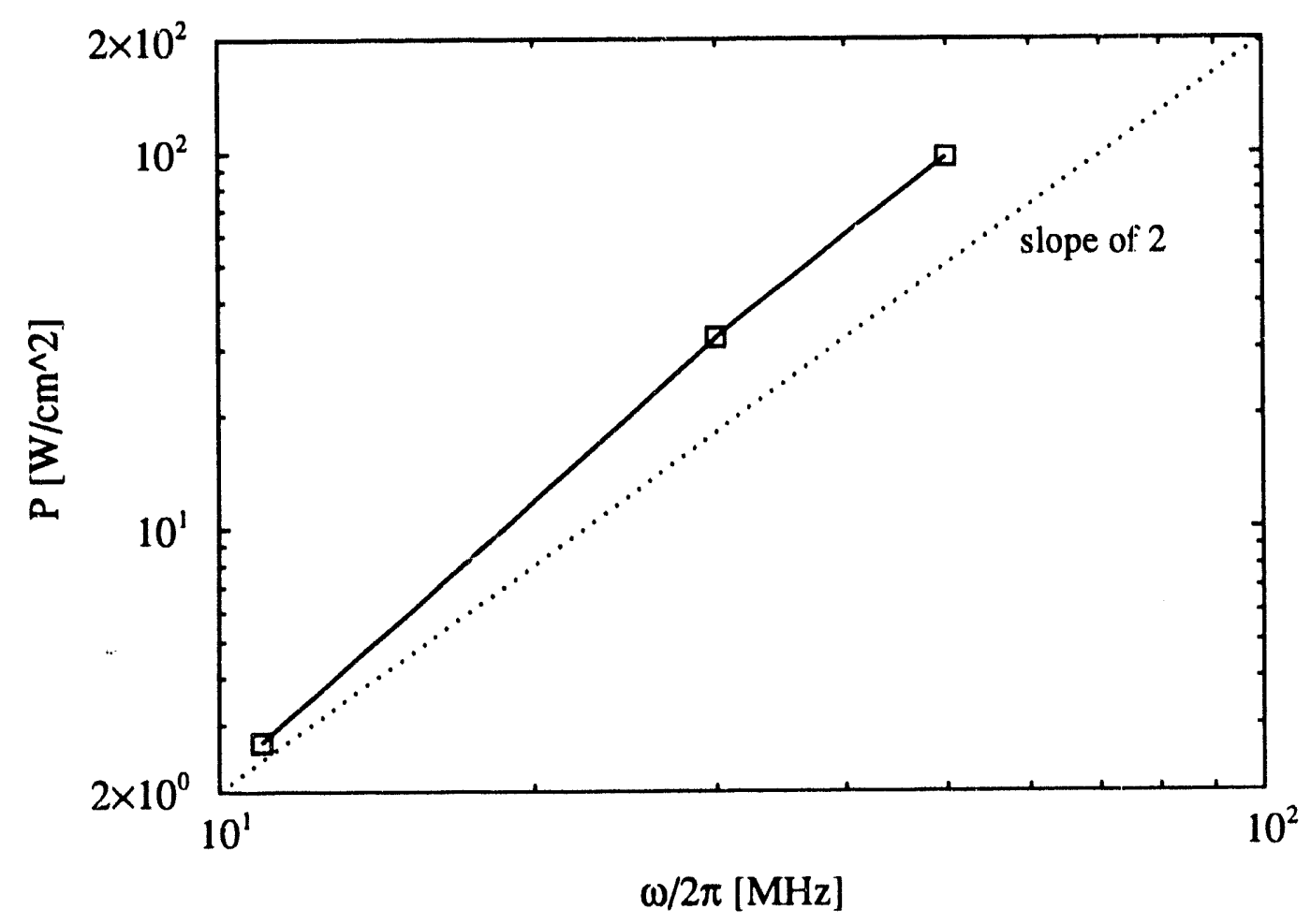

Figure 8: 


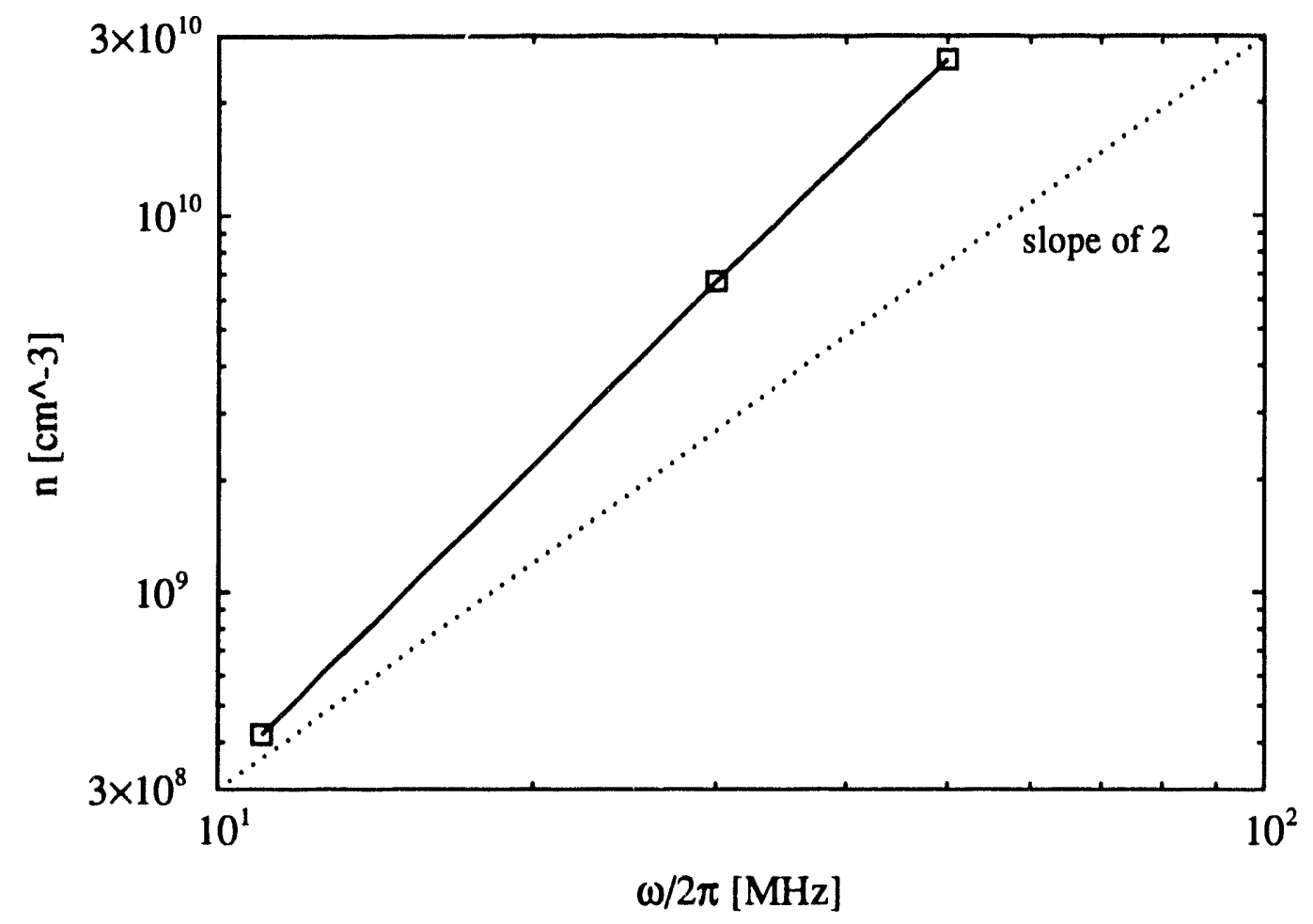

Figure 9: 


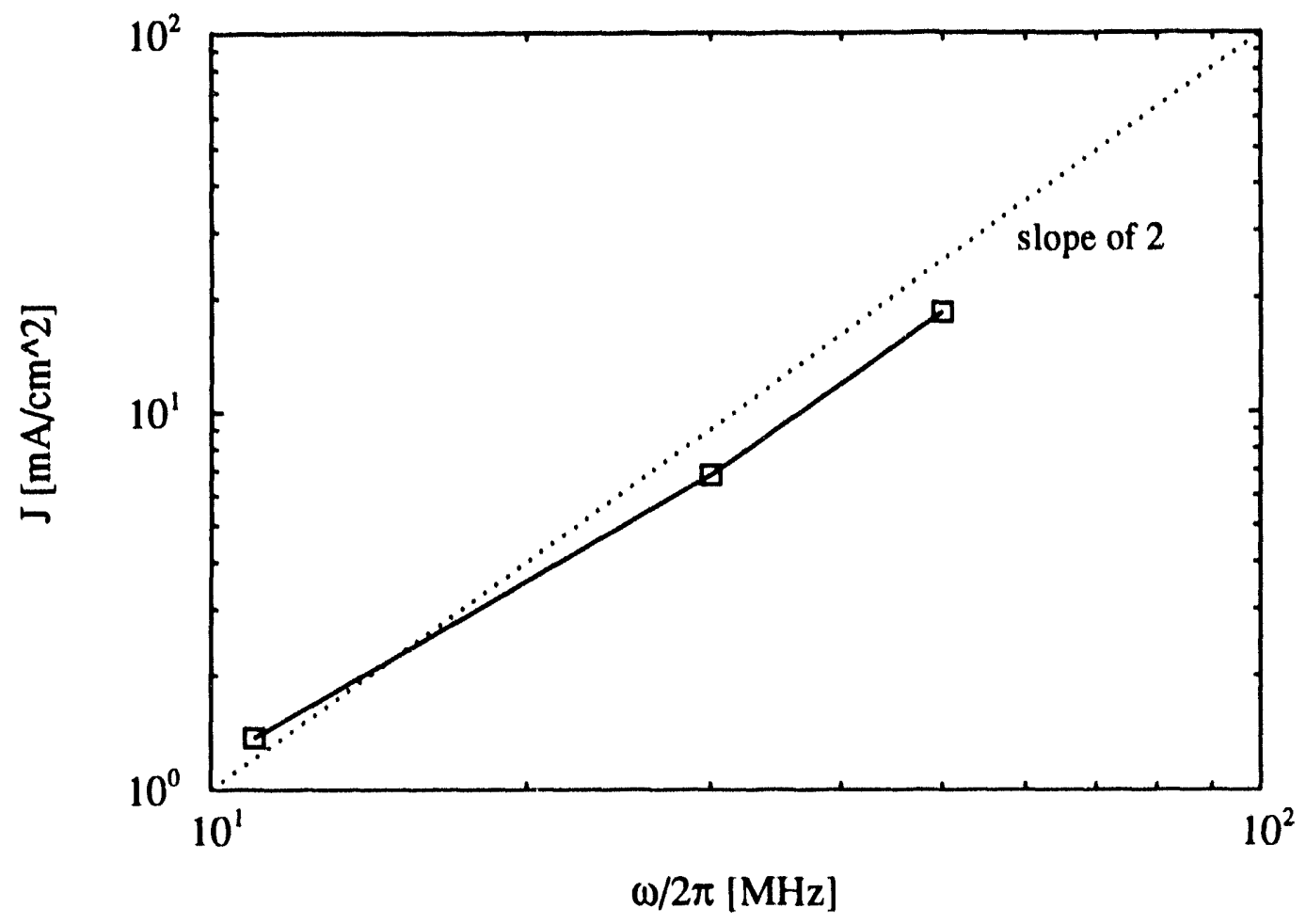

Figure 10: 




Figure 11: 


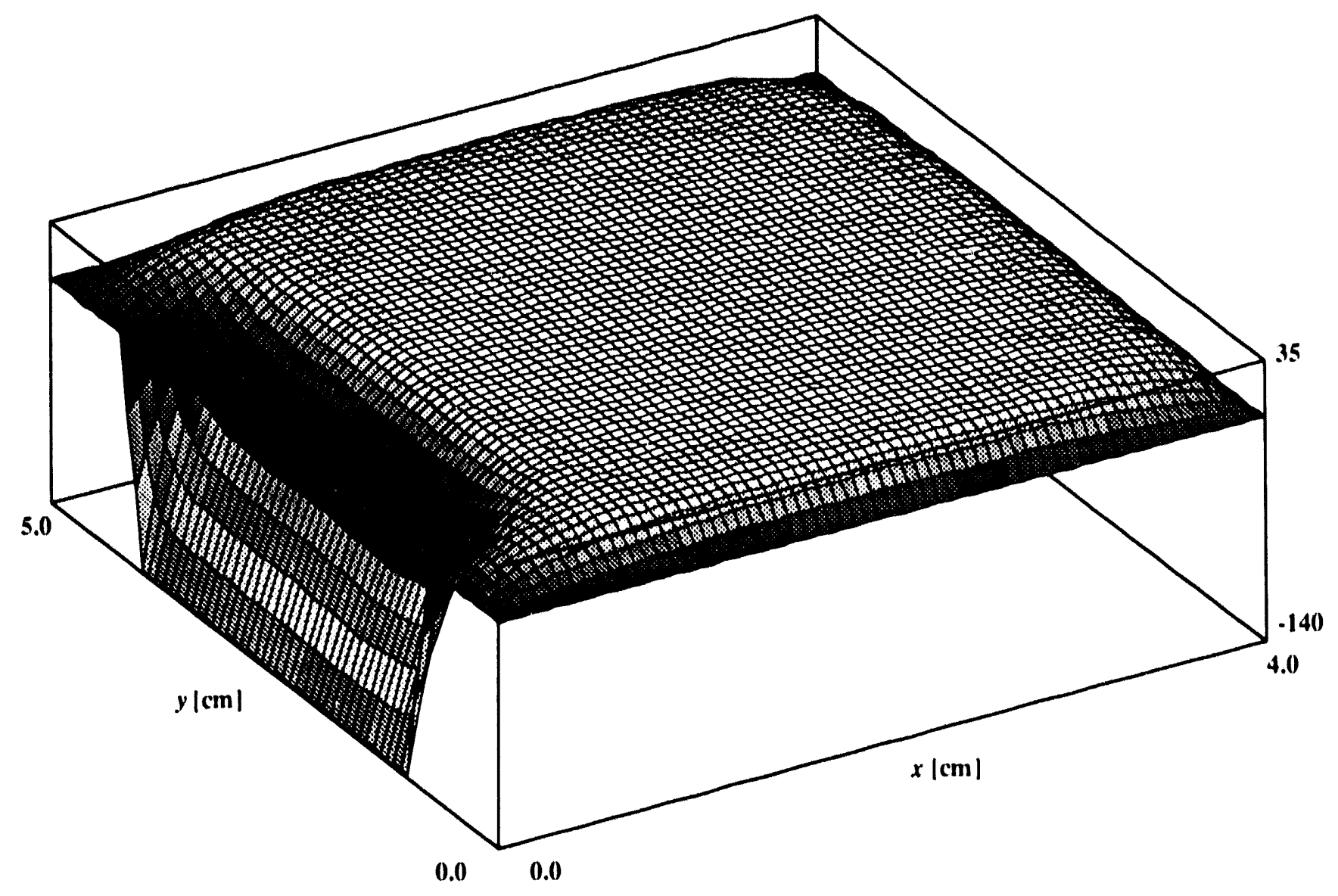

Figure 12: 


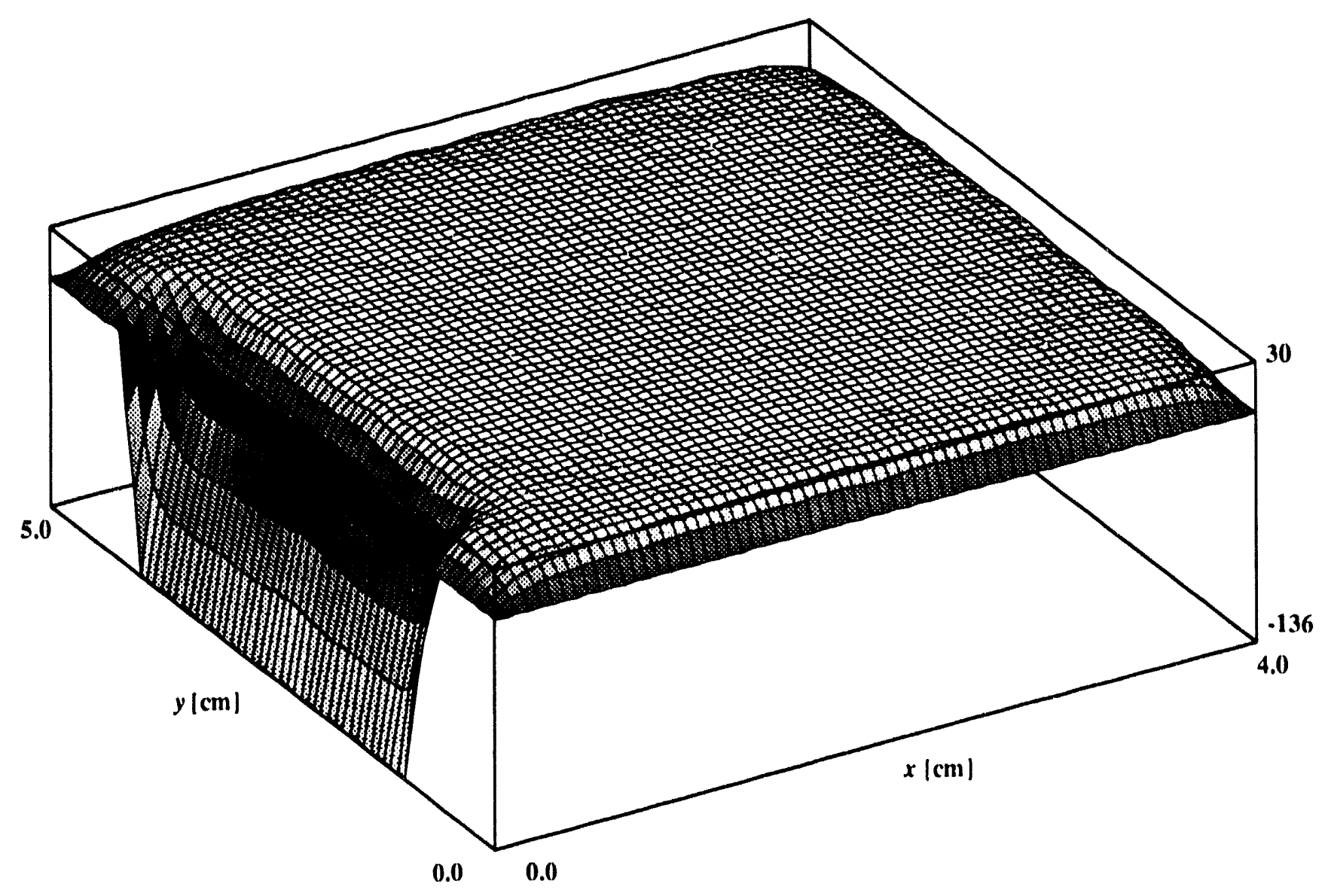

Figure 13: 

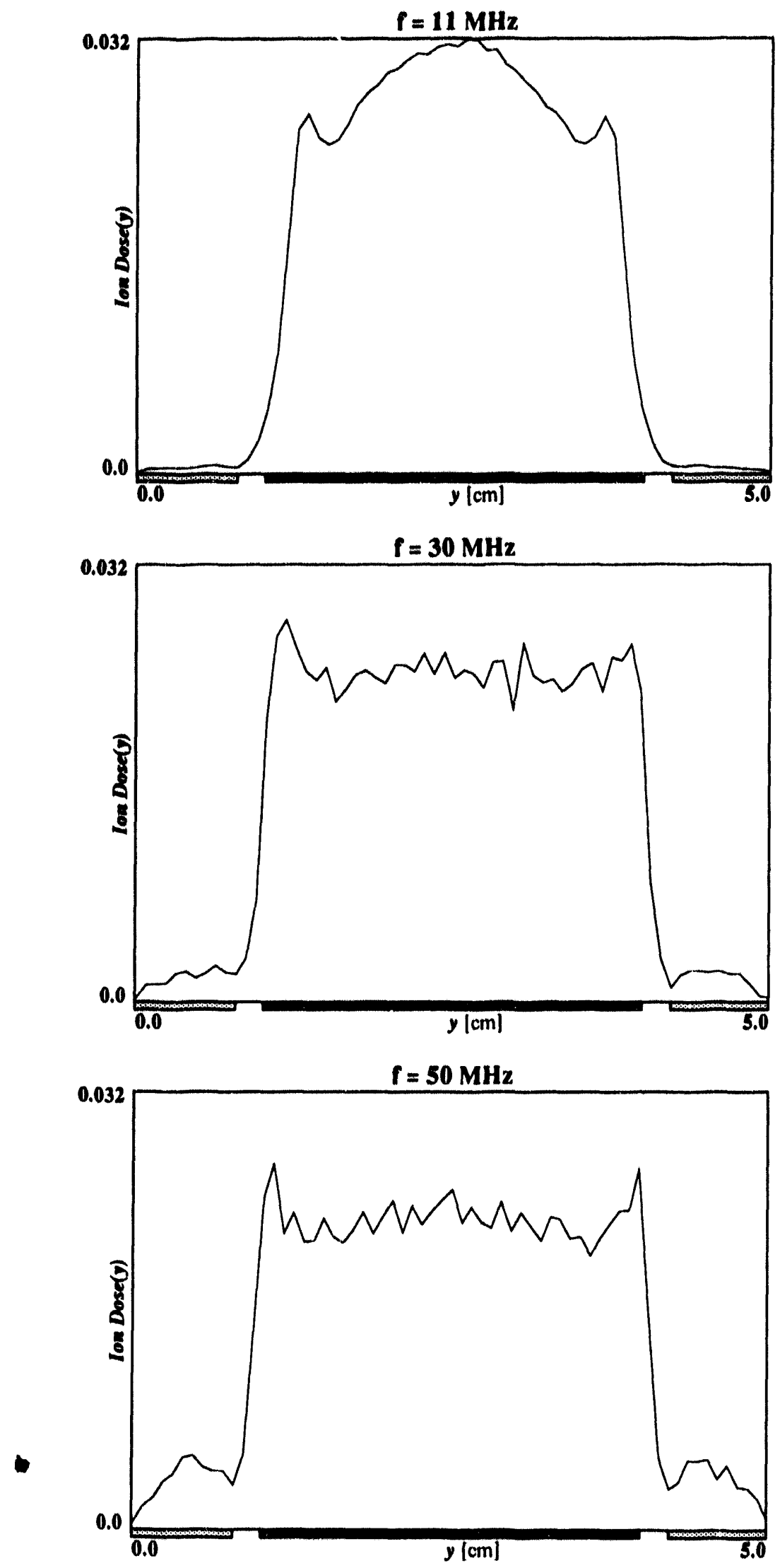

Figure 14: 


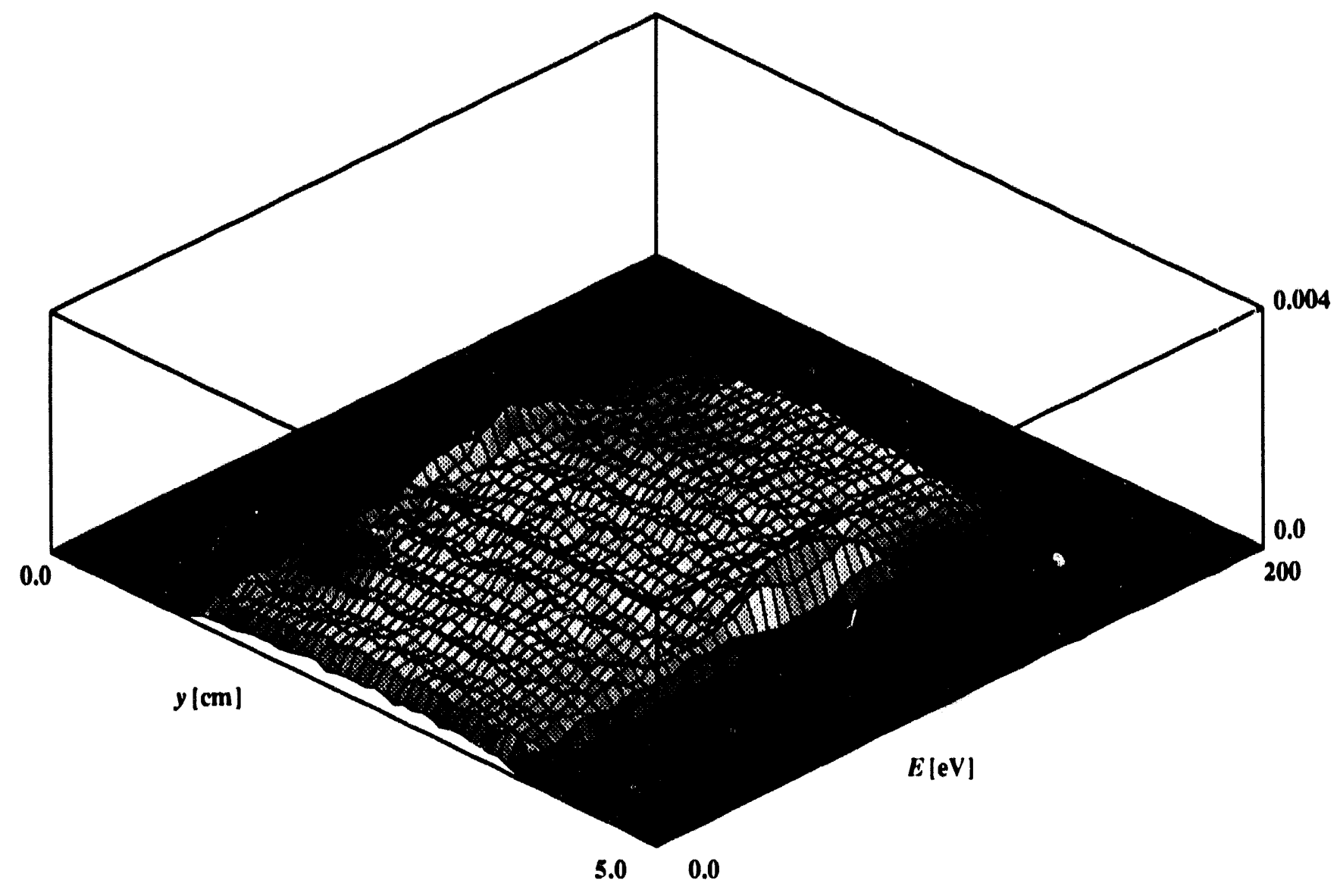

Figure 15: 


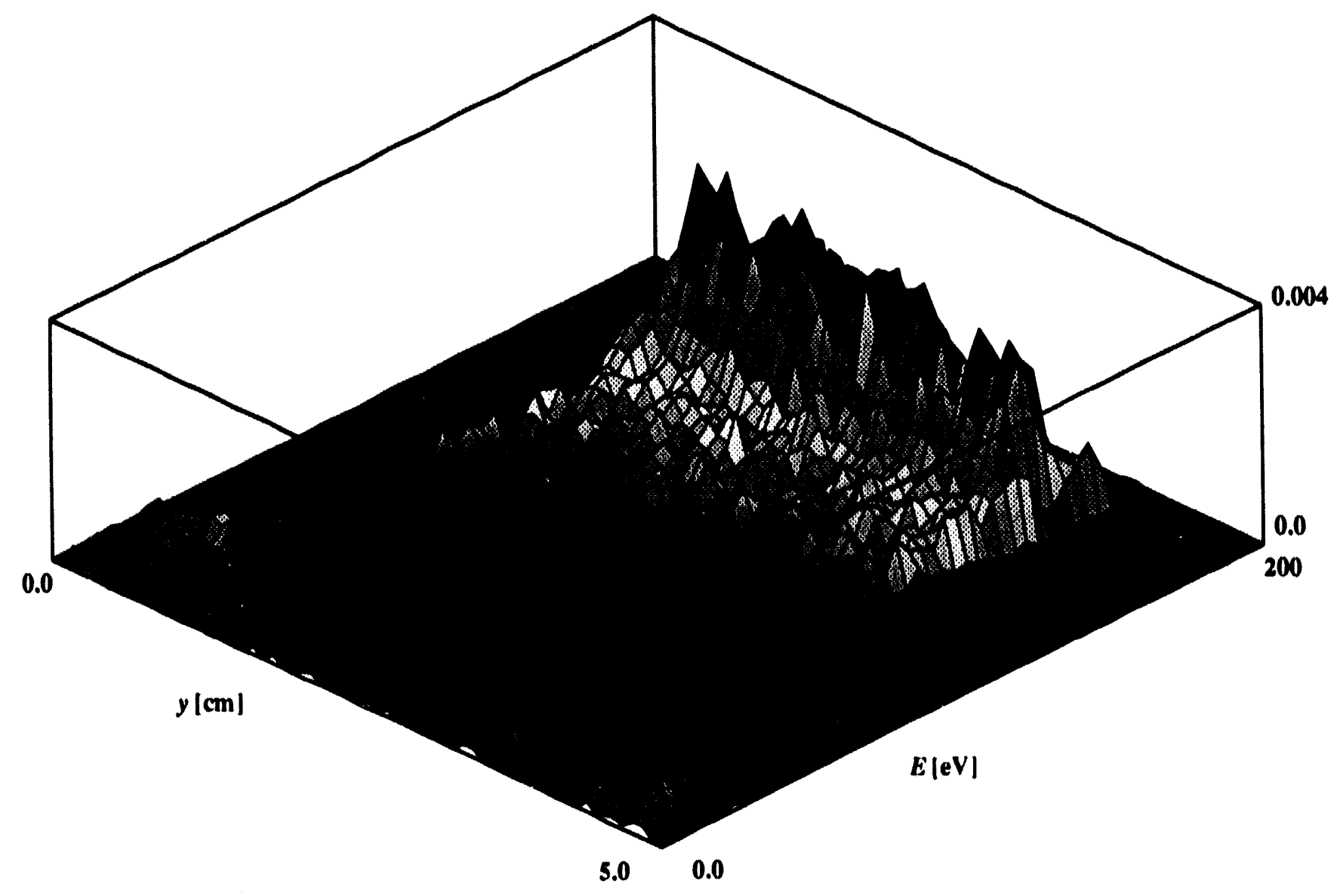

1

Figure 16: 


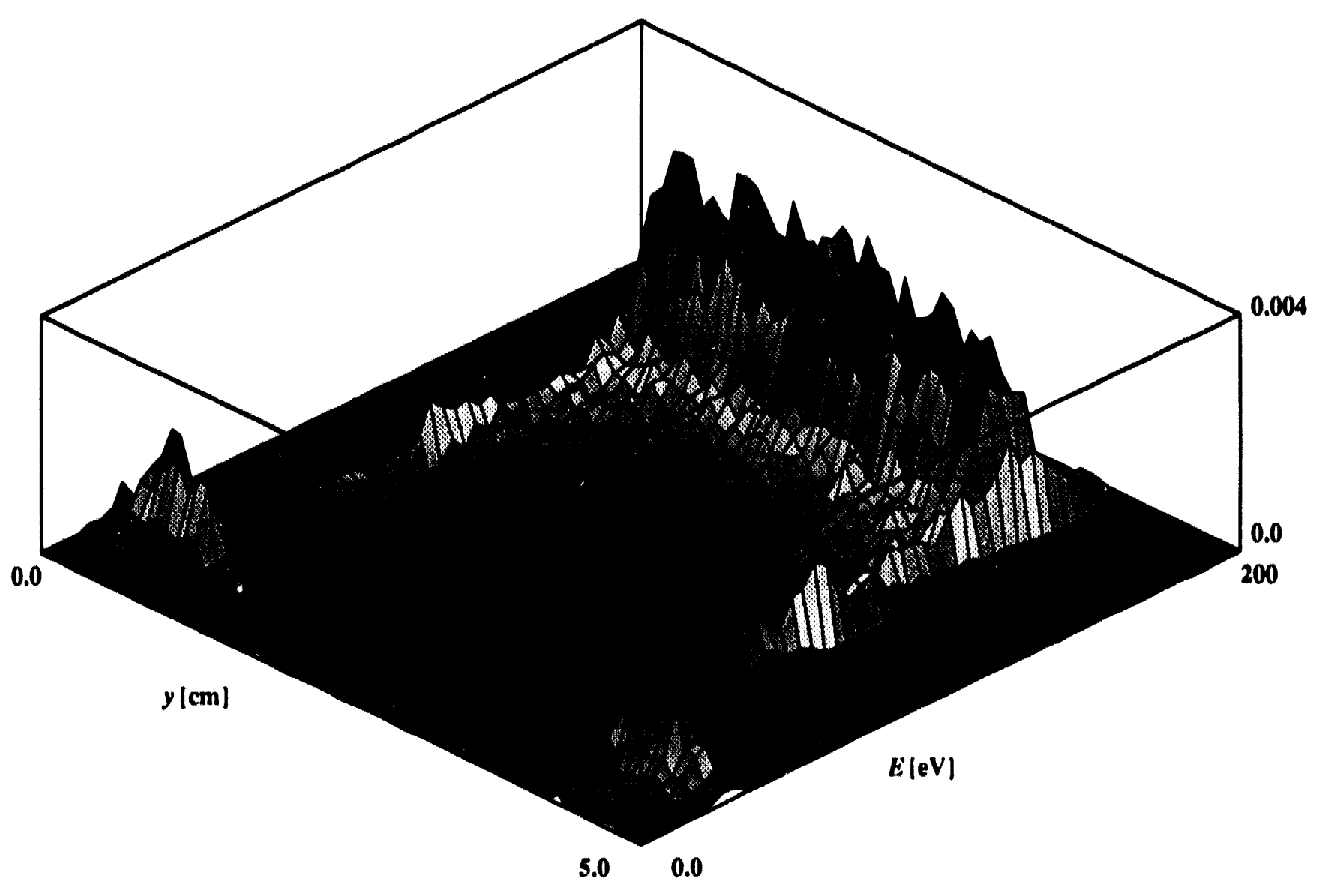

Figure 17: 

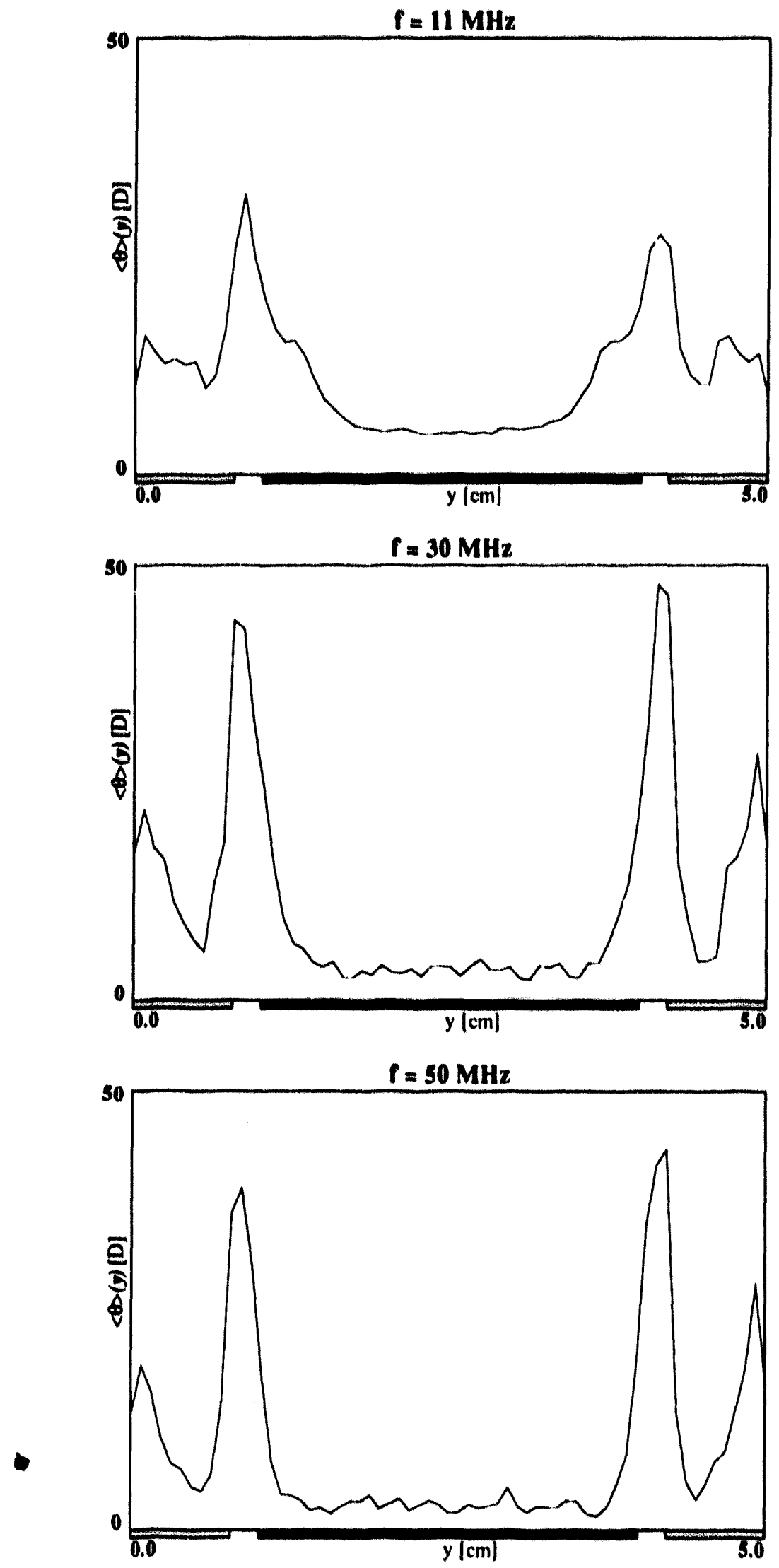

Figure 18: 

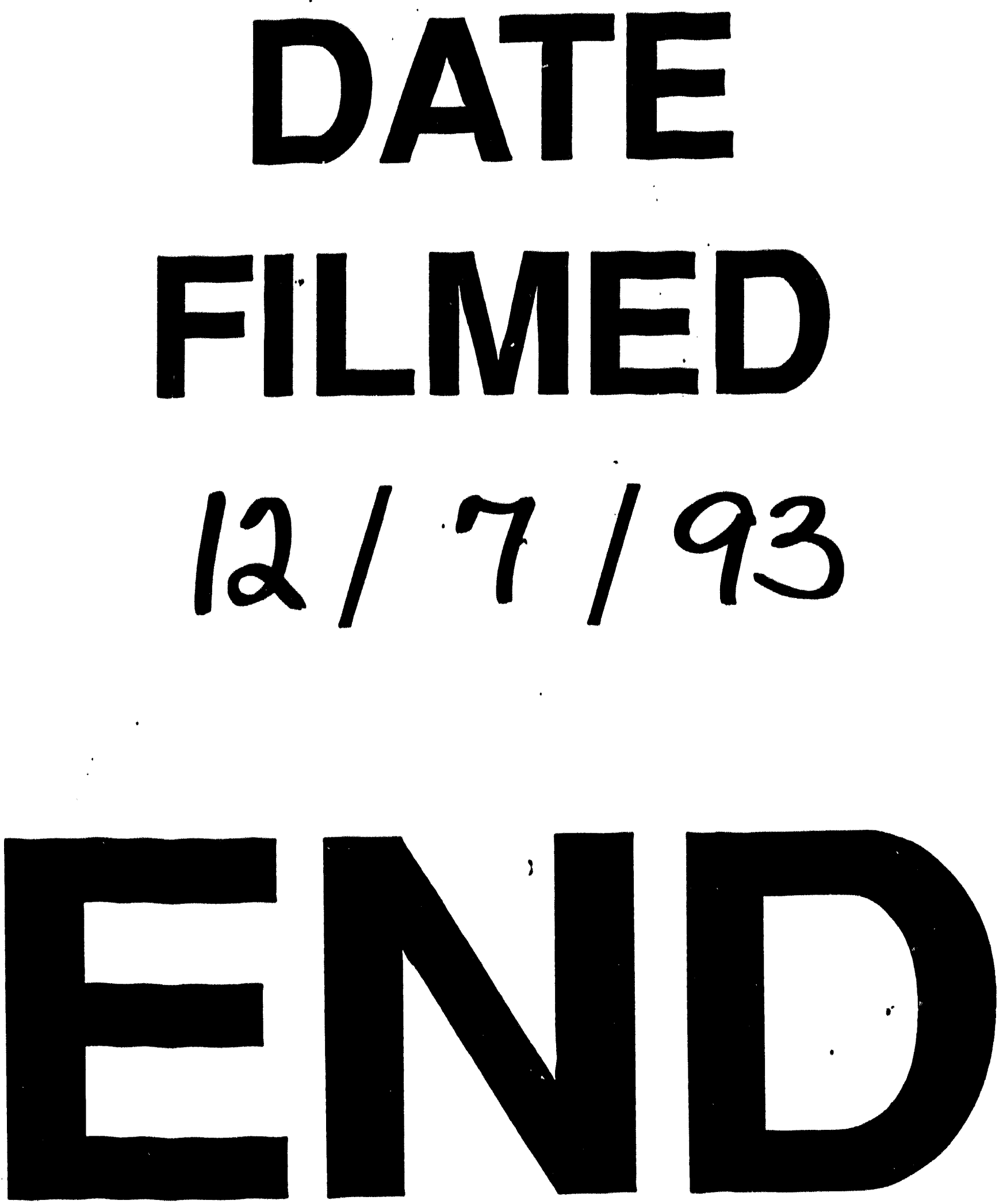
